Review

\title{
Health Benefits of Green Tea in Obesity
}

Mario F. Tecce ${ }^{1}$, Walter Milano ${ }^{2}$, Anna Capasso ${ }^{1, *}$

1. Department of Pharmacy, University of Salerno, Fisciano (Salerno), Italy; E-Mails: tecce@unisa.it; annacap@unisa.it; pharmacologyunisa@gmail.com

2. U.O.S.D. Eating Disorder Unit - Mental Health Department ASL Napoli 2 Nord, Napoli, Italy; EMail:wamilano@tin.it

* Correspondence: Anna Capasso; E-Mails: annacap@unisa.it; pharmacologyunisa@gmail.com

Academic Editor: Rafat A. Siddiqui

Special Issue: Managing Obesity through Nutraceuticals

OBM Integrative and Complementary Medicine 2020 , volume 5 , issue 3

doi:10.21926/obm.icm.2003038
Received: June 26, 2020

Accepted: August 17, 2020

Published: August 30, 2020

\begin{abstract}
Adipocytes play a central role in maintaining lipid homeostasis and energy balance by storing triacylglycerols (TAGs) or releasing free fatty acids in response to changes in energy requirements. Obesity is a major risk factor for many diseases, including diabetes, hypertension, and cardiovascular disease. Besides, morbidity and mortality associated with being overweight and obese are on the rise. The development of obesity is characterized by an increase in lipid cells due to mitogenesis and differentiation, which are regulated by genetic, endocrine, metabolic, neurological, pharmacological, environmental, and nutritional factors. Therefore, understanding the mechanism by which a given nutrient affects the mitogenesis of preadipocytes and their differentiation into adipocytes could help prevent the onset and progression of obesity, along with all its consequences in humans. It has been shown that nutraceuticals such as tea catechins and BCAAs (leucine, isoleucine, and valine) could be effective in preventing obesity. Green tea catechins (CTGs) are polyphenolic flavonoids, which were previously called vitamin P. CTGs, especially EGCGs, have been shown to reduce body weight and body fat. In support of its anti-obesity effect, some in vivo data have shown that EGCG or green tea extract containing EGCG reduces the absorption of food, lipids, TAGs, cholesterol, and leptins. Furthermore, it stimulates energy expenditure and fat oxidation, and ultimately increases the level of high-density lipoproteins and fecal excretion of lipids. EGCG regulates various enzymes linked to anabolism and lipid
\end{abstract} conditions of the Creative Commons by Attribution License, which permits unrestricted use, distribution, and reproduction in any medium or format, provided the original work is correctly cited. 
catabolism, such as acetyl-CoA carboxylase (ACC), fatty acid synthase, pancreatic lipase, gastric lipase, and lipoxygenase; it also reduces the increase in insulin (INS) and TAG during the differentiation period of nine days. Taken together, these observations, in vivo and in vitro, suggest that the EGCG contained in green tea can modulate the mitogenic, endocrine, and metabolic functions of fat cells. In this study, we report the biochemical and biological evidence indicating the benefits of green tea in obesity.

\section{Keywords}

Diet; green tea; nutraceuticals; obesity; overweight

\section{Introduction}

From an etiological point of view, obesity has a multifactorial origin and is connected to modifiable risk factors (behaviors, lifestyle, and living conditions) and non-modifiable risk factors (genetic determinants) [1-5].

When the calorie intake exceeds calorie expenditure, a state of positive energy balance occurs, which increases body mass. A person can, therefore, become obese if, despite having normal energy expenditure, the calorie intake is excessive or if, despite feeding properly, he has a deficit in energy metabolism [6]. The complexity of the problem is determined by multilevel, interpersonal (family, social networks), community (school, workplaces, institutions), governmental influences (local, regional, national context, and policies). Even behavioral risk factors, including incorrect eating habits and sedentary lifestyle, which are often considered the main determinants of overweight and obesity, are strongly affected by complex collective dynamics involving large sectors of society: from families to schools, from health institutions to social organizations and mass media [7].

In parallel with the increase in the incidence of obesity and the progression of obese individuals toward higher BMI values, there is an increased risk of comorbidity. In Europe, overweight and obesity are responsible for about:

- $80 \%$ of cases of type 2 diabetes

- $55 \%$ of cases of high blood pressure

- $35 \%$ of cases of ischemic heart disease

All this translates into 1 million deaths and 12 million patients a year worldwide [8].

All the main obesity guidelines indicate that the first step of therapy is lifestyle modification through nutritional intervention, an increase in structured physical activity, and behavioral changes. However, long-term treatment is problematic and requires an integrated approach, which uses the tools available in a complementary way, making use of different professional skills, which share the same therapeutic objective [8]. It has now been established that to conquer the obesity epidemic; it is necessary to resort to various therapies (nutritional, cognitive-behavioral, pharmacological, and surgical) according to the individual patient [8]. The clinical goal must be to significantly decrease the bodyweight to significantly improve the risks associated with obesity, especially cardiovascular ones. However, if this strategy seems insufficient or ineffective, obese 
individuals can resort to drug therapy, taking into account that chronic diseases such as obesity must be managed, and treatment must be adapted to the individual patient [8].

Along with diet, physical activity (appropriate to the type of patient) and possibly also behavioral therapy must be combined. According to the American guidelines, the duration of drug therapy ranges from six months to one year for more complex cases. The use of surgical interventions is recommended only for extreme cases, for patients presenting acute obesity (with $\mathrm{BMI}>35-40$ ) and other comorbidities, who are at high risk of mortality, or who do not respond to other treatments. Besides, in recent years, there has been increasing use of natural remedies as adjuvants in the treatment of obesity, and green tea seems to be an effective remedy in the prevention of obesity [9].

\section{Green Tea: Biochemical and Biological Basis for Health Benefits in Obesity}

Many bioactive nutrients of vegetable origin present in the diet intervene in multiple cell signaling pathways. Some of these nutrients are currently investigated, while others have already been approved for their beneficial effects. They are named nutraceutical, a term formed by the combination of words "nutrition" and "pharmaceutical", which was coined by Stephen De Felice in 1989 [10] to simply designate any substance considered a food (or part of a food) and can provide health benefits. These nutrients are chemically different molecules, which can act as molecular targets in specific tumorigenesis processes - proliferation, invasion, angiogenesis, and metastasis [10].

Modern medical research has confirmed that drinking tea regularly brings numerous health benefits. This is due to the presence of polyphenols, in particular (-) - epigallocatechin-3-gallate [11]. In China, tea is generally believed to prolong and improve people's lives. Among the types of tea, green tea, white tea, yellow tea, and Oolong are considered the healthiest as the type of processing to obtain these teas allows them to retain healthy substances [11].

Adipocytes play a central role in maintaining lipid homeostasis and energy balance, accumulating triacylglycerols (TAGs), or releasing free fatty acids, in response to changes in energy needs. Obesity is an important risk factor for a range of ailments, including diabetes, hypertension, and cardiovascular disease. The prevalence of morbidity and mortality associated with obesity has been increasing. The development of obesity is characterized by an increase in lipid cells due to mitogenesis and differentiation, which are regulated by genetic, endocrine, metabolic, neurological, pharmacological, environmental, and nutritional factors. As a result, understanding the mechanism by which a given nutrient affects preadipocyte mitogenesis and their differentiation into adipocytes could help prevent the onset and progression of obesity along with its consequences [12].

Also, based on the data from experimental animal models, the World Cancer Research Fund and American Institute for Cancer Research published a report in 2007 on the causal relationship between high body fat and an increased risk of CRC (colorectal cancer). Meta-analysis studies also revealed that the level of CRC risk was greater among obese men compared to non-obese men [13]. Studies have shown that nutraceuticals such as tea catechins and BCAAs (leucine, isoleucine, and valine) could be effective in preventing both colorectal and liver-related carcinogenesis and obesity [14]. 


\section{EGCG Reduces Body Fat}

Green tea catechins (CTG) are polyphenolic flavonoids, which were previously called vitamin P. CTGs, especially EGCGs, in addition to reducing the incidence of tumors, collagenopathies, neurodegenerative diseases induced by oxidative stress and streptozotocin-induced diabetes, are shown to reduce body weight and body fat [15]. In support of this anti-obesity effect, some in vivo data have shown that EGCG or EGCG-containing green tea extract reduces the absorption of food, lipids, TAGs, cholesterol, and leptins. Also, it stimulates energy expenditure and oxidation of fats and finally increases the level of high-density lipoproteins and fecal excretion of lipids [15]. EGCG regulates various enzymes linked to lipid anabolism and catabolism, such as acetyl-CoA carboxylase (ACC), fatty acid synthase, pancreatic lipase, gastric lipase, and lipoxygenase; it also reduces the increase in insulin (INS) and TAG in a 9-day differentiation period. Taken together, these in vivo and in vitro observations suggest that EGCG contained in green tea is capable of modulating the mitogenic, endocrine, and metabolic functions of fat cells [16].

\section{Effects of EGCG on Cell Viability in 3T3-L1 Cultures}

A study was conducted to evaluate the anti-adipogenic action of EGCG and to understand how this molecule acts to reduce the expression of adipocyte protein markers and their target genes during the differentiation of 3T3-L1 cells. 3T3-L1 is a cell line derived from mouse 3T3 cells that are used in biological research on adipose tissue because they differentiate into cells with a phenotype similar to adipocytes. The specific objective of this study was to establish the molecular mechanisms underlying the mitigating effect of EGCG on cell viability, adipocyte differentiation, and lipid accumulation [16].

It is well known that obesity results in increased size (hypertrophy) and number (hyperplasia) of adipocytes [17]. CTG treatment can decrease both the cell number and cell viability [18]. A significant reduction in cell viability was immediately observed after treatment, at different concentrations and time, with three specific catechins, in particular-EC, EGC, and EGCG. The results showed that with the increase in time and concentration, EGCG was the most effective of the three. In the study, the variation in cell viability was measured using a representative concentration of EGCG $(100 \mu \mathrm{M})$, with particular attention to the duration of treatment and differentiation of adipocytes: the differentiation was induced by exposing the cells to a culture medium consisting of $10 \%$ fetal bovine serum, $10 \mu \mathrm{g} / \mathrm{mL}$ of INS (insulin), $1 \mu \mathrm{M}$ of dexamethasone, and $0.5 \mu \mathrm{M}$ of 3-isobutyl-1-methylxanthines for $72 \mathrm{~h}$. It has been shown that short-term treatment ( 3 and five days) did not affect the viability of 3T3-L1 cells, while long-term treatment $(6,7$, and 12 days) decreased cell viability. These results suggest that treatment with EGCG during differentiation of adipocytes is necessary and sufficient to prevent the accumulation of mature adipocytes [16].

\section{Effects of EGCG on Adipocyte Differentiation}

Also, during exercise, there is increased activity of AMPK (AMP-activate protein kinase) in the muscle. AMPK is a protein kinase that modulates cellular metabolism by phosphorylation of different enzymes. It checks the energy status and signals when the level is low. Its activation takes place when there is a cellular energy deficit, just like during physical exercise, where the 
activated AMPK blocks the production of adipocytes, promoting the increase in energy consumption and weight loss.

Activated AMPK promotes a series of a metabolic cascade of events such as:

- increase in fat burning processes;

- increase in the number of mitochondria, so that more energy (ATP) can be obtained from fat;

- inhibition of gluconeogenetic processes, to encourage the use of fat as energy;

- reduction in insulin release, which would otherwise promote the accumulation of additional fat.

Activation of AMPK by EGCG appears to be involved in the inhibition of differentiation of 3T3L1 cells, grown in normal medium, and then exposed to EGCG in the differentiation medium. The percentage of activation of AMPK and the phosphorylation of its acetyl-CoA carboxylase (P-ACC) substrate was subsequently measured using western blot. As a result, the phosphorylation of both AMPK (P-AMPK) and ACC (P-ACC) has significantly increased in a time-dependent manner [16]. Therefore, in this study, we demonstrated how the inhibitory effect of EGCG on the differentiation of adipocytes is dependent on the activation of AMPK.

\section{Effect of EGCG on the Accumulation of Lipids during the Differentiation of Adipocytes}

The anti-adipogenic effect of EGCG on 3T3-L1 cells was assessed based on the duration of treatment in hormone-induced adipogenesis (insulin increases the differentiation of adipocytes and the accumulation of lipids) [19]. In this study, a high percentage of 3T3-L1 cells, treated with adipogenic cocktails of INS, dexamethasone, and 3-isobutyl-1-methylxanthine, acquired the typical morphology of differentiated fat cells. At this point, the lipid accumulations were detected by staining with ORO (Oil Red O) of the differentiated 3T3-L1 cells (with untreated induction) compared to the control (without untreated induction). However, ORO staining and TAG content were attenuated by longer duration of EGCG treatment, while no reduction in lipid and TAG accumulation was observed after short-term treatment (3 days). Interestingly, the TAG content was not affected by EGCG treatment even on day 5, suggesting that longer treatment $(6.7$, and 12 days) is important for the anti-adipogenic effect of EGCG. These results confirm the antiadipogenic effect of EGCG on 3T3-L1 cells, also the inhibitory effect on hormone-induced adipogenesis [16].

\section{Green Tea Extract Contributes to the Prevention of NAFLD}

It is well known that a diet rich in saturated fat (HFD) and low in dietary fiber, can lead to obesity. Obesity, just like any other systemic and multifactorial disease, causes more damage than simple adipocyte hypertrophy [20]. By nonalcoholic hepatic steatosis (NAFLD), we mean nothing more than the hepatic manifestation of obesity which, will be the main cause of liver cirrhosis reducible by transplant in the next 20 years [21].

The liver is a vital organ for maintaining body homeostasis and to prevent and combat the onset of NAFLD. With the increase in visceral adiposity, there is an increase in the supply of nonesterified fatty acids (NEFA) to the liver. Studies have shown that the fraction of NEFA delivered to the liver by visceral fat is proportionally related to the area of visceral fat, and it is about $5-10 \%$ in normal-weight subjects and $20-30 \%$ in obese subjects. In the liver, NEFA is both oxidized and esterified to form triglycerides (TAG): the latter is thus stored in the cytosol before being 
incorporated into VLDL and then secreted. Now, when TAG production exceeds NEFA oxidation and VLDL secretion, TAGs accumulate in the liver, resulting in the development of NAFLD [22]. In another study, the level of NEFA was increased in the experimental groups, and, as we will see, the subsequent treatment through supplementation of green tea extract was able to reduce it, contributing to the prevention of the development of NAFLD [23].

\section{Experimental Study on Mice Fed with HFD}

The decaffeinated green tea extract can restore a normal hepatic metabolic profile and mitigate the effects induced by a high-fat diet (HFD), thereby preventing nonalcoholic fatty liver disease in mice. In a study by Santamarina et al. [23], the mice were maintained on a control (CD) or HFD diet for 16 weeks by supplementing this diet with water or green tea extract (50 $\mathrm{mg} / \mathrm{kg} / \mathrm{die}$ ) rich in EGCG. During the treatment, the increase in body mass, the level of adiponectin in the serum, and the lipid profile were measured; also, the expression of the pathway of the AMPK protein in the liver was measured. These experiments showed that mice fed a supplement of decaffeinated green tea extract benefited best from the antiadipogenic effect. The animals were divided into four experimental groups according to the type of diet (CD or HFD) and the type of supplement (decaffeinated green tea extract or water):

- $\mathrm{CD}+$ water $=\mathrm{CW}$ group

- $C D+$ decaffeinated green tea extract $=$ CE group

- $\mathrm{HFD}+$ water $=\mathrm{HFW}$ group

- HFD + decaffeinated green tea extract = HFE group

From week 4, weight gain in the CE group was less than that in the CW group; from the 8th week, the weight acquired in the HFW group was higher than that of the $\mathrm{CW}$ group. Furthermore, the weight gain in the HFE group was lower than in the HFW group. The lipid content in the HFW group was higher than the other groups [23]. The serum analysis showed a decrease in the level of non-esterified fatty acids and an increase in the level of adiponectin in the CE and HFE groups compared to their corresponding placebo group. An increase in the secretion of VLDL-TG was observed in the HFW group compared to the CW and HFE groups while in the HFE group there was an increase in the expression of proteins, AdipoR2, SIRT1, pLKB1, and pAMPK, which explains the reduced expression of ACC, FAS, SREBP-1, and ChREBP in this group. These results indicate that the effects of decaffeinated green tea extract may be related to the activation of AMPK via LKB1 (liver serine/threonine kinase and main activator of AMPK) in the liver of HFD-fed mice [23].

\section{Green Tea Extract in the Regulation of Lipogenesis}

LKB1 phosphorylation appears to be necessary for AMPK activation; the role of adiponectin in the activation of LKB1 is controversial where one study demonstrated its stimulation [24], while the other one did not [25]. The complex formed by LKB1 and AMPK has a key role in regulating the metabolism of liver fatty acids: this complex is activated by phosphorylation. Several molecules activate LKB1 in the liver: one of them is SIRT1. Activated AMPK (phosphorylated) can modulate lipogenesis, and also its phosphorylation leads to the phosphorylation and inactivation of acetylCoA carboxylase (ACC), which is an important regulatory enzyme in the synthesis of fatty acids [26]. ACC catalyzes the conversion of acetyl-CoA to malonyl-CoA, which becomes a substrate for fatty acid synthase (FAS), an enzyme involved in the synthesis of fatty acids. The inhibition of ACC 
by AMPK, therefore, reduces the substrate flow for FAS, leading to a decrease in its activity. Moreover, AMPK is a negative regulator of SREBP-1c (sterol element-binding protein) and ChREBP (carbohydrate response element-binding protein), important transcription factors that activate lipogenic genes: this means that the increase in AMPK phosphorylation also determines a decrease in the level of nuclear SREBP 1 -c and ChREBP $[27,28]$.

Studies have shown that a single dose of green tea extract can increase the level of liver AMPK and the phosphorylation of LKB1, manifesting the powerful effects of green tea on these molecules. The extract could reduce the risk of obesity and have concrete protective effects against liver damage [29]. Stimulation of AMPK phosphorylation, which in turn inhibits ACC and FAS activity, could reduce the accumulation of liver triglycerides. Considering the lack of studies on the effect of decaffeinated green tea extract on the activation of AMPK via LKB1, we aimed to study the effects of green tea supplements on the activation of enzymes and factors related to liver lipogenesis, in conjunction with the secretion of VLDL-TG in HFD-fed mice [23].

\section{EGCG increases AMPK level}

In recent years, several authors have described that green tea polyphenols and isolated EGCG are effective in increasing the level of adiponectin in serum and the expression of its receptor proteins (AdipoR1 and AdipoR2) in humans and mice fed with HFD [30]. The expression of the adiponectin gene is mainly regulated by the nuclear receptor PPAR-y (peroxisome proliferatoractivated receptor): this recognizes and activates specific transcription factors that regulate gene expression. PPARs bind to specific regions of DNA called peroxisome proliferator response elements (PPRE), located in the promoter of genes subject to regulation. PPAR-y receptors are mainly expressed in adipose tissue, but also in the skeletal muscle, liver, heart, and kidney and regulate adipogenesis and lipid metabolism. They also regulate the expression of hormones or cytokines secreted by the adipocyte, such as leptin, adiponectin, and TNF-alpha. Thus, researchers have shown that green tea polyphenols can increase the expression of PPAR-y by reducing its phosphorylation [31]. Based on these results, it was found that green tea extract is also responsible for the increase in the level of adiponectin and AdipoR2, which in turn mediate the activation of SIRT1 protein [23].

Based on this hypothesis that adiponectin, through its receptor more abundant in the liver (AdipoR2), can stimulate the activation of SIRT1 in the HFE group, where SIRT1 itself, seems to have activated AMPK through an LKB1-dependent pathway. When activated, LKB1 migrates from the nucleus to the cytoplasm of the hepatocytes, where it induces phosphorylation of the $\alpha A M P K$ subunit, the main culprit of AMPK activity in the liver of rodents [32]. Therefore, activated AMPK can keep the forms SREBP1C and ChREBP phosphorylated in the cytoplasm and prevent them from reaching the nucleus. ChREBP and SREBP 1-c are transcription factors that regulate the expression of liver enzymes involved in lipogenesis, and they can bind to the transcription promoter genes of lipogenic proteins such as ACC and FAS. Both, ChREBP and SREBP 1-c remain in an inactive form in the cytoplasm, connected to a phosphate group: their dephosphorylated (active) form has, however, demonstrated the ability to move to the nucleus and stimulate the synthesis of lipogenic enzymes. Consequently, the AMPK activating cascade resulted in the reduction of the expression of these enzymes in the liver of the subjects of the HFE group compared to the HFW group [23]. 
Activated AMPK also allowed the simultaneous reduction of ACC and FAS: when phosphorylated, ACC is in its inactive form, and we have observed that supplementation with green tea extract has increased the PACC level as well as reduced the level of FAS, indicating a further element that allowed the reduction of the synthesis of fatty acids from the path of lipogenesis [23].

Overall, these results indicate that green tea extract supplementation has been able to improve liver metabolism and reduce the absorption of NEFA in the liver; this led to a decrease in the hepatic secretion of VLDL-TG, decreased lipid synthesis, and increased adiponectin in HFD-fed mice. It has been observed that the integration of green tea extract has also been shown to stimulate the activation of AMPK via LKB1 through adiponectin, in HFD-fed mice, and to regulate essential enzymes involved in the path of lipogenesis in the liver. In all these respects, it can certainly contribute to the prevention and treatment of NAFLD.

\section{Green Tea Protects Blood Vessels}

Green tea can intervene in the protection of blood vessels. In the late 1960s, scientists from the University of California compared the level of arteriosclerosis in the arteries of the brain and coronary arteries of about 300 western coffee drinkers and 100 Chinese tea drinkers based on values measured following autopsies performed during a decade. Compared to coffee drinkers, only two-thirds of tea drinkers had coronary artery damage, and only a third of brain artery damage. To study this phenomenon more closely, animal guinea pigs were fed with high-fat foods for three months so that they develop arteriosclerosis, and some of them were also given green tea along with drinking water. After three months, the blood vessels of these animals were less compromised than those of the animals who had only water. The scientists discovered that arteriosclerosis is combated with tea if it is taken at the same time or immediately after a high-fat meal. The Japanese and Chinese habit of drinking green tea during meals is therefore completely justified. In the meantime, it has been established that epigallocatechin gallate contained in the tea is the only compound responsible for reducing the cholesterol level. In the early 1980s, a study was conducted in Japan to verify whether green tea exerted a preventive effect on cardiovascular and liver diseases: in this regard, more than 1300 men were interviewed and examined; blood tests performed during this study indicated that those who drank lots of green tea had a much lower total cholesterol rate, resulting in a lower risk of arteriosclerosis. However, the most sensational discovery was that green tea could "distinguish" useful (HDL) and harmful (LDL) cholesterol: the rate of "good" cholesterol in the blood was increased in those who consumed the green tea regularly [33]

\section{Green Tea Modulates the Gut Microbiota}

Nowadays, a new thought is emerging due to the recent scientific discoveries that intestinal microbiota is directly responsible for the management of body weight. Many studies have shown that microbiota balance is a decisive element in ensuring the correct intestinal absorption of macronutrients. Metagenomic studies in obese patients have highlighted how obesity is associated with an imbalance in bacterial flora, with the proliferation of potentially pathogenic bacteria [34]. The alteration of the microbiota can affect the metabolism and energy homeostasis and is involved in the control of body weight through the extraction of an additional amount of 
calories from ingested food [35]. The mechanism is linked to the extraction of energy from food components such as polysaccharides (cellulose, hemicellulose, non-digestible starch, pectins, gums) which are processed by bacterial enzymes with the production of monosaccharides (subsequently absorbed) and short-chain fatty acids (SCFA, mainly acetate, butyrate, and propionate) that participate in the breakdown of fats in the liver, through the expression of some key enzymes such as acetyl-CoA carboxylase (ACC) and fatty acid synthase (FAS). The conversion of fermentable dietary fibers into SCFAs provides additional energy for the host, suggesting the possibility to promote obesity in this way [36]. In the context of digestive processes, we can make a rough distinction between fermentative flora that can digest starch and other polysaccharides, and putrefactive flora involved in putrefaction, anaerobic metabolization of peptides and proteins (elastin and collagen from food sources, pancreatic enzymes, exfoliated epithelial cells, and lysed bacteria), which determines the production of SCFA and potentially toxic substances, including ammonia, amines, phenols, thiols, and indoles [35]. The intestinal epithelium is covered by a double layer of mucus, internal and external, secreted by the goblet cells, and filled with mucins. These are glycosylated proteins that allow the intestinal mucus to perform a lubricating, trophic, and protective action against the mucosal epithelial cells. Its concentration strictly depends on a dynamic and delicate balance between synthesis, secretion, and degradation of the mucins that constitute it.

A recent study demonstrated the efficacy of a diet based on $2 \%$ green tea extract on mice [37]. Specifically, green tea suppressed inflammation and improved the health of the intestinal bacterial flora, which are the two key factors in preventing obesity. To demonstrate this, the researchers selected two groups of male mice; the first group was fed on a normal diet, the second group a high-fat one. To half of the members of each group, green tea extract was supplemented in the food. During the 8-week experimental period, the body weight and body mass index, insulin resistance, and other indicators of intestinal health, such as permeability, the composition of the bacterial population, and level of inflammation, were measured. It was found that half of the mice fed on a fat diet along with green tea gained less than $20 \%$ of weight than those fed on a fat diet without tea. In parallel, inflammation and insulin resistance dropped. Therefore, it is interesting to see how the mice treated with tea had a more impermeable and healthier intestine. On the contrary, the permeable intestine, also called "leaky gut," allows bacterial fragments and digestive residues to pass into the blood more easily, inducing generalized inflammation throughout the body. Finally, the healthiest species of the gut bacteria population were found in green tea fed mice. These results were also found in mice fed with tea and normal diet, in more modest percentages, than those fed on a fat diet. This confirms that lifestyle changes are especially useful for those who are ill [37]. Mice fed on a high-fat diet, and green tea gained about 20 percent less weight and had lower insulin resistance than mice fed on an identical diet without tea. The amount of green tea in the experiment would be equivalent to about 10 cups of green tea per person. A daily consumption value would not be far from the usual consumption in some areas of the world. This study provides evidence that green tea encourages the growth of good gut bacteria, and leads to several benefits that significantly reduce the risk of obesity [37]. 


\section{Conclusions}

In conclusion, the habitual consumption of green tea could prove to be an effective method to prevent obesity over time. Besides, green tea extracts can improve the physiological profile of the organism at certain doses. However, it is still too early to affirm the therapeutic activity of green tea extracts in the treatment of obesity, but some in vitro studies have shown that EGCG, the main polyphenolic component of green tea, is an efficient molecule for several molecular targets. Therefore, the discovery of EGCG as an inhibitor of multiple signaling pathways which, unlike specific inhibitors of single targets could reduce drug resistance by hindering the activation of alternative signaling pathways, constituting a step forward toward the introduction of a new drug, capable of acting against many important diseases such as obesity.

Concrete results have also been identified in the treatment of diseases associated with obesity: by reducing the number and activity of lipid cells. EGCG can reduce the obesity "factor" and all the pathologies, including diabetes, hypertension, and nonalcoholic hepatic steatosis (NAFLD), also demonstrating excellent prospects on its ability to reduce the absorption of lipids and stimulate energy expenditure. Several in vitro studies have shown that EGCG reduces cell viability, lipid differentiation, and accumulation through the activation of AMPK kinase and the consequent phosphorylation and inactivation of acetyl-CoA carboxylase, an important regulator of synthesis of fatty acids. The only limitation is that a dose of $100 \mu \mathrm{M}$ of EGCG did not give any result on shortterm lipid reduction, but a prolonged treatment for 12 days reduced cell viability and accumulation of lipids, in particular, triglycerides and cholesterol in adipocytic cells.

However, decaffeinated green tea extract that is essentially rich in EGCG restored a normal hepatic metabolic profile in mice fed with a high-fat diet (HFD), preventing the development of nonalcoholic liver steatosis, which is identified as a hepatic manifestation of obesity. Furthermore, serum analysis showed a reduction in the levels of non-esterified fatty acids and an increase in adiponectin, which is responsible for the activation of AMPK via LKB1 in the liver. Therefore, this signaling process, as in obesity, reduces the activation of acetyl-CoA carboxylase. Also, in this case, the duration of treatment influenced the study result, ensuring a reduction in body weight and body fat accumulation in mice after 16 weeks of treatment with $50 \mathrm{mg} / \mathrm{kg} / \mathrm{day}$.

Overall, green tea extract has proven to be an excellent compound for the evaluation of new drug formulations and supplements, which are already sold in the market. However, tea contains two molecules - caffeine and theine. Although present in small quantities and its effect is reduced in association with polyphenols present in tea, it is mainly responsible for side effects and drug interactions. Finally, we can conclude by saying that how much enjoying a good cup of tea can only help your health.

\section{Author Contributions}

Study concept and design: Mario F. Tecce and Anna Capasso.

Acquisition, analysis, or interpretation of data: All authors.

Drafting of the manuscript: All authors.

Critical revision of the manuscript for important intellectual content: All authors.

Study supervision: Mario F. Tecce and Anna Capasso. 


\section{Competing Interests}

The authors have declared that no competing interests exist.

\section{References}

1. OECD. The heavy burden of obesity: The economics of prevention. Paris: OECD Publishing; 2019.

2. GBD 2017 Diet Collaborators. Health effects of dietary risks in 195 countries, 1990-2017: A systematic analysis for the Global Burden of Disease Study 2017. Lancet. 2019; 393: 19581972. doi: 10.1016/S0140-6736(19)30041-8.

3. Pednekar MS, Jóźwiak J, Kolsteren P, Giwercman A, Van-Herck K, Bettiol H, et al. Worldwide trends in body-mass index, underweight, overweight, and obesity from 1975 to 2016: A pooled analysis of 2416 population-based measurement studies in 128.9 million children, adolescents, and adults. Lancet. 2017; 390: 2627-2642. doi: 10.1016/S0140-6736(17)32129-3.

4. Yang W, Kelly T, He J. Genetic epidemiology of obesity. Epidemiol Rev. 2007; 29: 49-61.

5. Swinburn BA, Sacks G, Hall KD, McPherson K, Finegood DT, Moodie ML, et al. The global obesity pandemic: Shaped by global drivers and local environments. Lancet. 2011; 378: 804814.

6. Magni P. Obesity: Pathophysiology and new diagnostic perspectives, page 5. Kaleidoscope Italia.

7. Lang T, Rayner G. Overcoming policy cacophony on obesity: An ecological public health framework for policymakers. Obes Rev. 2007; 8: 165-181.

8. Italian Standards for the Treatment of Obesity 2016-2017 Italian Obesity Society (SIO) and Italian Association of Dietetics and Clinical Nutrition (ADI).

9. Prasanth MI, Sivamaruthi BS, Chaiyasut C, Tencomnao T. A review of the role of green tea (camellia sinensis) in antiphotoaging, stress resistance, neuroprotection, and autophagy. Nutrients. 2019; 11: 474.

10. DeFelice SL. The NutraCeutical revolution: Fueling a powerful, new international market. 1989.

11. Ferruzzi MG. The influence of beverage composition on delivery of phenolic compounds from coffee and tea. Physiol Behav. 2010; 100: 33-41.

12. Unger RH, Zhou YT. Lipotoxicity of beta-cells in obesity and in other causes of fatty acid spillover. Diabetes. 2001; 50: S118.

13. Renehan AG, Tyson M, Egger M, Heller RF, Zwahlen M. Body mass index and incidence of cancer: A systematic review and meta-analysis of prospective observational studies. Lancet. 2008; 371: 569-578.

14. Shimizu M, Kubota M, Tanaka T, Moriwaki H. Nutraceutical approach for preventing obesityrelated colorectal and liver carcinogenesis. Int J Mol Sci. 2012; 13: 579-595.

15. Liao S, Kao YH, Hiipakka RA. Green tea: Biochemical and biological basis for health benefits. Vitam Horm. 2011; 62: 1-94.

16. Moon HS, Chung CS, Lee HG, Kim TG, Choi YJ, Cho CS. Inhibitory effect of epigallocatechin-3gallate on lipid accumulation of 3T3-L1 cells. Obesity. 2007; 15: 2571-2582. 
17. Wolfram S, Wang Y, Thielecke F. Anti-obesity effects of green tea: From bedside to bench. Mol Nutr Food Res. 2006; 50: 176-187.

18. Hung PF, Wu BT, Chen HC, Chen YH. Antimitogenic effect of green tea epigallocatechin gallate on 3T3-L1 preadipocytes depends on the ERK and Cdk2 pathways. Am J Physiol Cell Physiol. 2005; 288: C1094-C1108.

19. Scavo LM, Karas M, Murray M, Leroith D. Insulin-like growth factor stimulates both cell growth and lipogenesis during differentiation of human mesenchymal stem cells into adipocytes. J Clin Endocrinol Metab. 2004; 89: 3543-3553.

20. Gregor MF, Hotamisligil GS. Inflammatory mechanisms in obesity. Annu Rev Immunol. 2011; 29: 415-445.

21. Charlton MR, Burns JM, Pedersen R, Watt KD, Heimbach JK, Dierkhising R. Frequency and outcomes of liver transplantation for nonalcoholic steatohepatitis in the United States. Gastroenterology. 2011; 141: 1249-1253.

22. Stanhope KL, Havel PJ. Fructose consumption: Potential mechanisms for its effects to increase visceral adiposity and induce dyslipidemia and insulin resistance. Curr Opin Lipidol. 2008; 19: 16-24.

23. Santamarina AB, Oliveira JL, Silva FP, Carnier J, Mennitti LV, Santana AA, et al. Green tea extract rich in epigallocatechin-3-gallate prevents fatty liver by AMPK activation via LKB1 in mice fed a high-fat. PloS ONE. 2015; 10: e0141227.

24. Blagih J, Krawczyk CM, Jones RG. LKB1 and AMPK: Central regulators of lymphocyte metabolism and function. Immunol Rev. 2012; 249: 59-71.

25. Shearn CT, Smathers RL, Jiang H, Orlicky DJ, Maclean KN, Petersen DR. Increased dietary fat contributes to dysregulation of the LKB1/AMPK pathway and increased damage in a mouse model of early stage ethanol mediated steatosis. J Nutr Biochem. 2013; 1: 1436-1445.

26. Hasenour CM, Berglund ED, Wasserman DH. Emerging role of AMP-activated protein kinase in endocrine control of metabolism in the liver. Mol Cell Endocrinol. 2013; 366: 152-162.

27. Seo HY, Kim MK, Jung YA, Jang BK, Yoo EK, Park KG, et al. Clusterin decreases hepatic SREBP1c expression and lipid accumulation. Endocrinology. 2013; 154: 1722-1730.

28. lizuka K. Recent progress on the role of ChREBP in glucose and lipid metabolism. Endocrinology. 2013; 60: 543-555.

29. Banerjee S, Ghoshal S, Porter TD. Phosphorylation of hepatic AMP activated protein kinase and liver kinase B1 is increased after a single oral dose of green tea extract to mice. Nutr Res. 2012; 32: 985-990.

30. Wang S, Moustaid-Moussa N, Chen L, Mo H, Shastri A, Su R, et al. Novel insights of dietary polyphenols and obesity. J Nutr Biochem. 2014; 25: 1-18.

31. Tian C, Ye X, Zhang R, Long J, Ren W, Ding S, et al. Green tea polyphenols reduced fat deposits in high fat-fed rats via erk1/2-PPARY-adiponectin pathway. PLoS ONE. 2013; 8: e53796.

32. Cantó C, Auwerx J. PGC-1alpha, SIRT1 and AMPK, an energy sensing network that controls energy expenditure. Curr Opin Lipidol. 2009; 20: 98-105.

33. Hu HF, Zerbst M. Il tè verde. Milano: Tecniche Nuove; 1999.

34. Dao MC, Clement K. Gut microbiota and obesity: Concepts relevant to clinical care. Eur J Int Med. 2018; 48: 18-24.

35. Angelakis $E$, Armougom F, Million M, Raoult D. The relationship between gut microbiota and weight gain in humans. Future Microbiol. 2012; 7: 91-109. 
36. Krajmalnik-Brown R, Ilhan ZE, Kang DW, DiBaise JK. Effects of gut microbes on nutrient absorption and energy regulation. Nutr Clin Pract. 2012; 27: 201-214.

37. Chung E, Campise SN, Joiner HE, Tomison MD, Kaur G, Dufour JM, et al. Effect of annattoextracted tocotrienols and green tea polyphenols on glucose homeostasis and skeletal muscle metabolism in obese male mice. J Nutr Biochem. 2019; 67: 36-43.

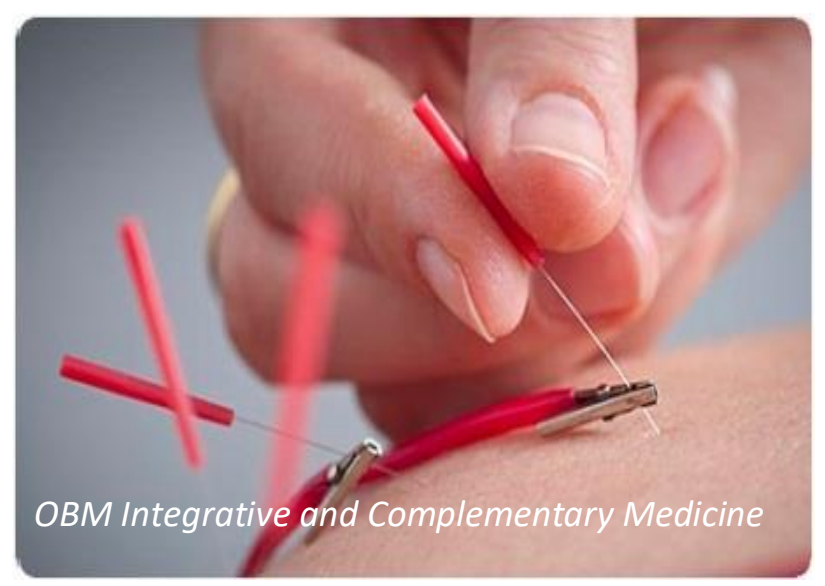

Enjoy OBM Integrative and Complementary Medicine by:

1. Submitting a manuscript

2. Joining in volunteer reviewer bank

3. Joining Editorial Board

4. Guest editing a special issue

For more details, please visit:

http://www.lidsen.com/journals/icm 